Article

\title{
Candidacidal Activity of a Novel Killer Toxin from Wickerhamomyces anomalus against Fluconazole-Susceptible and -Resistant Strains
}

\author{
Laura Giovati $^{1}{ }^{(\mathbb{D})}$, Claudia Santinoli $^{1}$, Elena Ferrari ${ }^{1}$, Tecla Ciociola ${ }^{1}{ }^{(\mathbb{D}}$, Elena Martin ${ }^{2}$, \\ Claudio Bandi ${ }^{2,3}$, Irene Ricci ${ }^{4}$, Sara Epis ${ }^{2,3}$ and Stefania Conti ${ }^{1, *}$ (iD \\ 1 Department of Medicine and Surgery, University of Parma, 43125 Parma, Italy; laura.giovati@unipr.it (L.G.); \\ claudia.santinoli@libero.it (C.S.); elena.ferrari@unipr.it (E.F.); tecla.ciociola@unipr.it (T.C.) \\ 2 Department of Biosciences, University of Milan, 20133 Milan, Italy; elena.martin@unimi.it (E.M.); \\ claudio.bandi@unimi.it (C.B.); sara.epis@unimi.it (S.E.) \\ 3 Pediatric Clinical Research Center Romeo and Enrica Invernizzi, Ospedale "Luigi Sacco", 20157 Milan, Italy \\ 4 School of Biosciences and Veterinary Medicine, University of Camerino, 62032 Camerino, Italy; \\ irene.ricci@unicam.it \\ * Correspondence: stefania.conti@unipr.it; Tel.: +39-0521-903492
}

Received: 1 December 2017; Accepted: 1 February 2018; Published: 3 February 2018

\begin{abstract}
The isolation and characterization from the sand fly Phlebotomus perniciosus of a Wickerhamomyces anomalus yeast strain $(W a 1 \mathrm{~F} 1)$ displaying the killer phenotype was recently reported. In the present work, the killer toxin (KT) produced by Wa1F1 was purified and characterized, and its antimicrobial activity in vitro was investigated against fluconazole- susceptible and -resistant clinical isolates and laboratory strains of Candida albicans and C. glabrata displaying known mutations. Wa1F1-KT showed a differential killing ability against different mutant strains of the same species. The results may be useful for the design of therapeutic molecules based on Wa1F1-KT and the study of yeast resistance mechanisms.
\end{abstract}

Keywords: Wickerhamomyces anomalus; yeast killer toxin; Candida albicans; Candida glabrata; antifungal resistance

Key Contribution: A novel killer toxin produced by the recently isolated Wickerhamomyces anomalus strain 1F1 showed a differential killing ability against fluconazole-susceptible and -resistant Candida clinical isolates and laboratory strains displaying known mutations.

\section{Introduction}

Yeast strains belonging to diverse species produce and secrete proteins or glycoproteins, known as killer toxins (KTs), that are lethal to susceptible strains [1,2]. This property, which offers a competitive advantage to self-immune killer yeasts in their ecological niches, has found several applications in the biological control of plant pathogens and spoiling yeasts in the food and fermentation industries [3]. In the medical field, KTs have been used for the biotyping of pathogenic microorganisms, in epidemiological studies, and for the identification of novel cellular targets in microbial cells and the development of new antimicrobials [4-6].

Some KTs, such as K1 and K28 from Saccharomyces cerevisiae, have a narrow spectrum of activity, limited to susceptible strains of the same species, while other KTs show a wide killing spectrum [2]. In particular, some Wickerhamomyces anomalus (formerly Pichia anomala) KTs proved to be active against a wide range of microorganisms, including other yeast species, filamentous fungi, bacteria, and protozoan parasites $[2,7,8]$. 
Killer strains of $W$. anomalus have been isolated from different sources, including plants and food products [9-11], arthropods such as the crab Portunus trituberculatus [12], and mosquitoes of the species Anopheles stephensi $[13,14]$. Recently, the isolation and characterization of a W. anomalus strain displaying the killer phenotype was reported from specimens of the sand fly Phlebotomus perniciosus [15].

The ability to inhibit harmful microorganisms in a variety of habitats and the wide killing spectrum of the produced KTs have prompted the use of $W$. anomalus as a bio-control agent $[7,16]$, since it could be classed as a low risk microorganism, rarely traced in human samples [17,18]. In addition, W. anomalus KTs activity against dermatophytes and pathogenic yeasts, especially Candida spp., led to the hypothesis that could be applied in medical mycology as alternative antifungal compounds [19-21].

Candida spp. are the most frequently isolated yeasts in clinical specimens. The frequency of invasive opportunistic fungal infections caused by species of this genus has significantly increased in recent years, particularly in immunosuppressed individuals and patients with indwelling medical devices [22]. Worldwide, the prevalent cause of invasive candidiasis remains C. albicans, although the epidemiology of candidal infections has gradually shifted towards non-albicans species, such as C. glabrata and C. krusei [22-24]. Increasing concern is rising in view of growing reports of resistance to antifungal drugs, with particular reference to resistance to azoles in non-albicans Candida species $[25,26]$.

In the aim of searching for new molecules, potentially effective against strains resistant to conventional antifungal drugs, we investigated the in vitro activity of a KT produced by the recently isolated W. anomalus strain 1F1 (Wa1F1-KT) [15] against both susceptible and azole-resistant clinical isolates as well as laboratory strains of $C$. albicans and C. glabrata displaying known mutations [27-31].

\section{Results}

\subsection{Wa1F1-KT Production}

The production of Wa1F1-KT by the strain W. anomalus $1 \mathrm{~F} 1$ was analyzed over time. The activity of the concentrated culture supernatant obtained at different time periods was determined against the reference C. lusitaniae strain on solid medium and quantified by Arbitrary Units/mL (1 Arbitrary Unit (AU) is defined as the amount of KT producing an inhibition zone of $1 \mathrm{~mm}^{2}$ ). As shown in Figure 1 , the results indicate that the activity of the Wa1F1-KT sample obtained after $24 \mathrm{~h}$ of incubation was low (559 AU/mL), although it increased after $48 \mathrm{~h}$ of incubation $(1815 \mathrm{AU} / \mathrm{mL})$, and reached a maximum after $72 \mathrm{~h}(2326 \mathrm{AU} / \mathrm{mL})$. The killing activity decreased steeply thereafter.

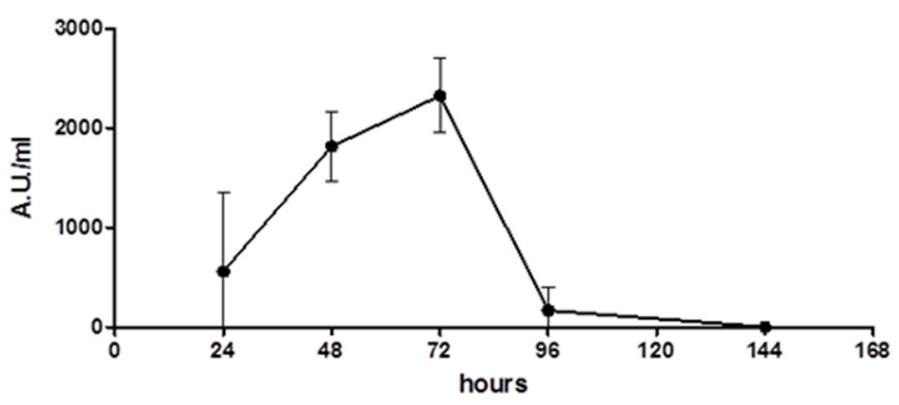

Figure 1. Time-course of Wa1F1-KT production by Wickerhamomyces anomalus 1F1. Overnight grown liquid seed cultures of the yeast were inoculated at $1 \%(v / v)$ into killer toxin (KT) production medium, then incubated at $20^{\circ} \mathrm{C}$ under shaking $(180 \mathrm{rpm})$. Samples were withdrawn at $24 \mathrm{~h}$ intervals, yeast cells were removed by centrifugation, and the filtered supernatants were concentrated 50-fold and assayed for their activity against the reference Candida lusitaniae strain grown on solid medium. Arbitrary Unit (AU): amount of KT which produces an inhibition zone of $1 \mathrm{~mm}^{2}$.

\subsection{Western Blot Analysis of Wa1F1-KT}

Western blot analysis was carried out on crude extracts from 72-h cultures of the W. anomalus strains 1F1 and ATCC 96603 (KT-producing, positive control strain). As a negative control, a crude 
extract from $W$. anomalus UM3 (KT-nonproducing strain) culture and 50-fold concentrated YPD medium were used. Bands were detected with $\mathrm{mAbKT} 4$, a monoclonal antibody directed against a KT produced by the reference ATCC 96603 strain (Wa96603-KT) and shown to cross-react with KTs produced by other W. anomalus strains and other killer yeasts $[14,32,33]$. The results showed that mAbKT4 reacts with high-molecular mass proteins in samples obtained from W. anomalus 1F1 and ATCC 96603, but not from controls (Figure 2). A single band of approximately $220 \mathrm{kDa}$ was revealed in extracts from the reference strain, while a single band with a lower molecular mass (approximately 160-170 kDa) was detected in the extract from 1F1 strain, indicating the secretion of KTs with a common epitope, although with some structural differences.

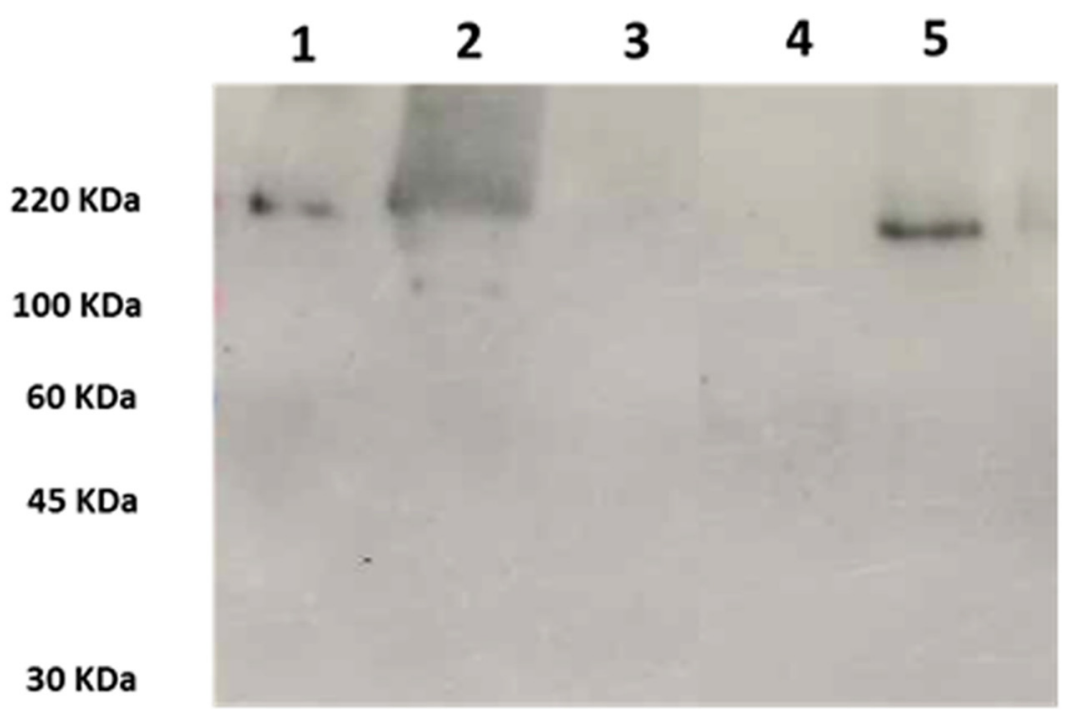

Figure 2. Western blot analysis using mAbKT4 to probe 50-fold concentrated crude extracts of (1) Wickerhamomyces anomalus ATCC 96603, $2 \mu \mathrm{L}$; (2) W. anomalus ATCC 96603, $5 \mu \mathrm{L}$; (3) W. anomalus $\mathrm{UM} 3,5 \mu \mathrm{L}$; (4) YPD medium, $5 \mu \mathrm{L}$; (5) W. anomalus 1F1, $5 \mu \mathrm{L}$. Molecular masses (kDa) are shown on the left.

\subsection{Characterization of Wa1F1-KT Using Size Exclusion Chromatography}

The concentrated extract from $W$. anomalus 1F1 culture was analyzed by size exclusion chromatography and eluted fractions were assayed for killing activity against the reference C. lusitaniae strain. In agreement with the immunoblot data, the results indicated the elution of active Wa1F1-KT in fractions 34-39 of the size exclusion chromatogram (Figure 3), corresponding to the highest molecular mass separation zone.

\subsection{Activity of Wa1F1-KT}

Wa1F1-KT was tested by an agar diffusion assay against the reference C. lusitaniae NEQAS 6208 strain and clinical isolates and laboratory strains of C. albicans and C. glabrata susceptible or resistant to fluconazole. The results showed that, when tested at $25{ }^{\circ} \mathrm{C}, \mathrm{Wa} 1 \mathrm{~F} 1-\mathrm{KT}$ was active towards both fluconazole-susceptible and -resistant strains of C. glabrata, with some quantitative differences (Table 1). Wa1F1-KT showed no effect against the reference C. albicans SC5314 strain and against two fluconazole-susceptible clinical isolates (DSY347 and DSY544) and the DSY544-derived fluconazole-resistant mutant strain (DSY775) of C. albicans. On the contrary, a good effect was detected against the DSY347-derived fluconazole-resistant mutant C. albicans DSY289 strain. No effect against any of the tested strains was detected when the assay was carried out at 30 and $37^{\circ} \mathrm{C}$. 
Table 1. Sensitivity of laboratory strains and clinical isolates of Candida spp. susceptible or resistant to fluconazole against killer toxin Wa1F1-KT.

\begin{tabular}{|c|c|c|}
\hline Strain & Features and Genotype & $\begin{array}{c}\text { Wa1F1-KT Sensitivity } \\
\text { (mm Growth Inhibition) a }\end{array}$ \\
\hline C. albicans SC5314 & Reference laboratory strain, FluS & 0 \\
\hline C. albicans DSY347 & FluS clinical strain [27] & 0 \\
\hline C. albicans DSY 289 & FluR, DSY347 ERG11: S405F, Y132H; TAC1: A736V [27,29] & 14 \\
\hline C. albicans DSY544 & FluS clinical strain [30] & 0 \\
\hline C. albicans DSY775 & FluR, DSY544 ERG11: G464S, TAC1: G980W [30] & 0 \\
\hline C. glabrata DSY562 & FluS clinical strain [28] & 13 \\
\hline C. glabrata DSY565 & FluR clinical strain [28] & 12 \\
\hline C. glabrata SFY93 & FluR, DSY562 pdr1 $\Delta[31]$ & 12 \\
\hline C. glabrata SFY105 & FluR, DSY562 pdr1 $\Delta-T 588 \mathrm{~A}[31]$ & 13 \\
\hline C. glabrata SFY115 & FluR, DSY562 pdr1 $\Delta-\mathrm{L} 280 \mathrm{~F}[31]$ & 13 \\
\hline C. glabrata SFY116 & FluR, DSY562 pdr1 $\Delta-P 822 L[31]$ & 13 \\
\hline C. lusitaniae NEQAS6208 & Reference laboratory strain, FluS & 17 \\
\hline
\end{tabular}

${ }^{\mathrm{a}}$ Diameter $(\mathrm{mm})$ of growth inhibition zone, mean values $( \pm 1 \mathrm{~mm})$ from four independent experiments. FluS, Fluconazole-susceptible; FluR, Fluconazole-resistant.

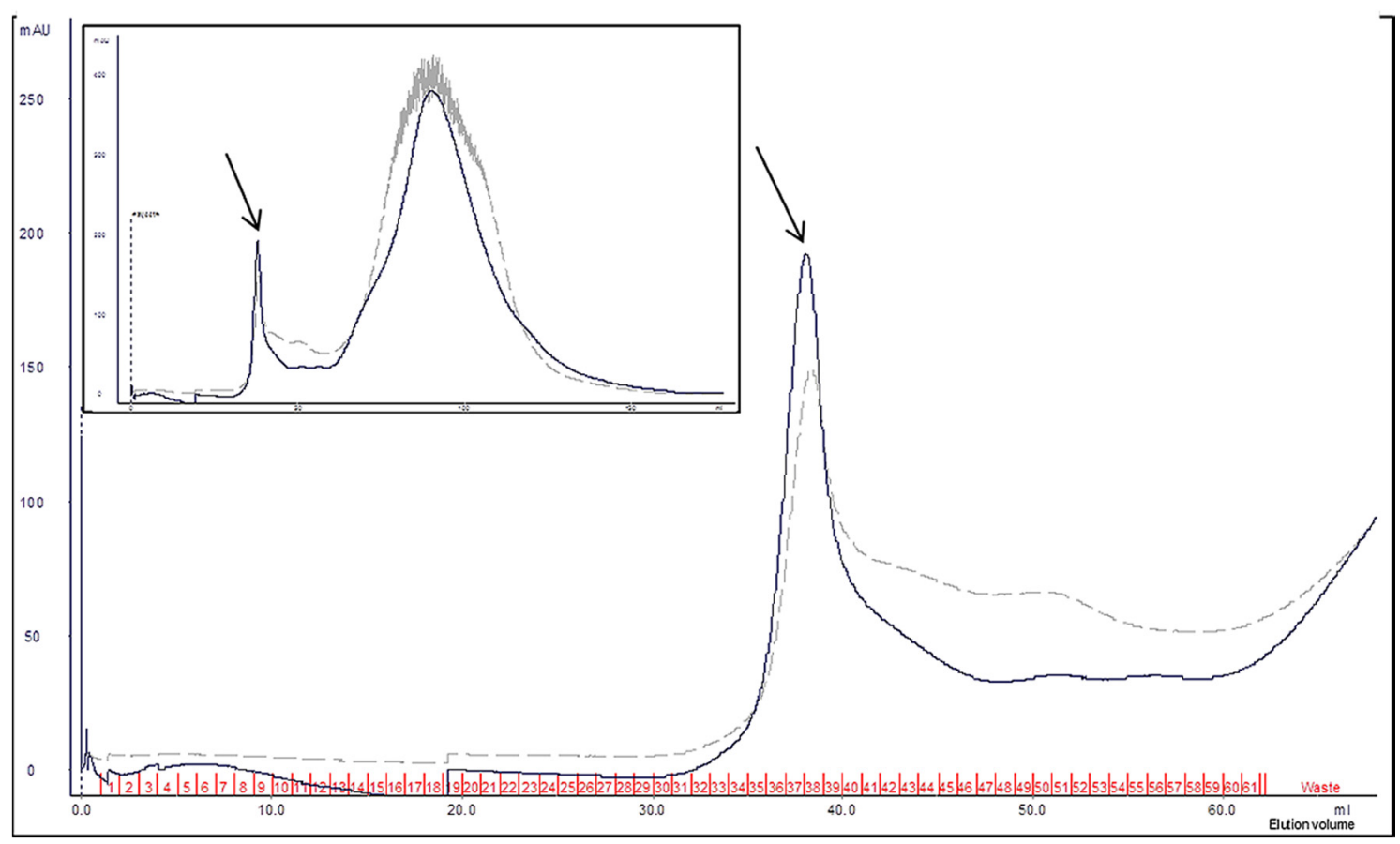

Figure 3. Wickerhamomyces anomalus 1F1 crude extract separation on HiPrep Sephacryl S-200 column. Partial chromatographic traces, displaying the active fractions (34-39), as determined by killing assay on Candida lusitaniae NEQAS 6208. Continuous and dashed lines refer to absorbance values (milli Absorbance Units, mAU) at $280 \mathrm{~nm}$ (mainly Trp absorbance) and $215 \mathrm{~nm}$ (peptide bond absorbance), respectively. Inset: complete chromatogram traces. The arrows indicate the elution peak of active fractions.

\subsection{Exo- $\beta-1,3-G l u c a n a s e$ Activity}

To investigate if Wa1F1-KT could act on susceptible yeast strains by hydrolyzing major cell wall components, its ability to digest the soluble $\beta$-1,3-glucan laminarin was assayed in comparison with Wa96603-KT, the KT produced by the Williopsis saturnus var. mrakii MUCL 41968 (Wm41968-KT), with known $\beta$-glucanase activity [34], and laminarinase. The results showed that all KTs could hydrolyze laminarin to an end product presenting similar relative mobility to the product of the laminarinase reaction (Figure 4). 

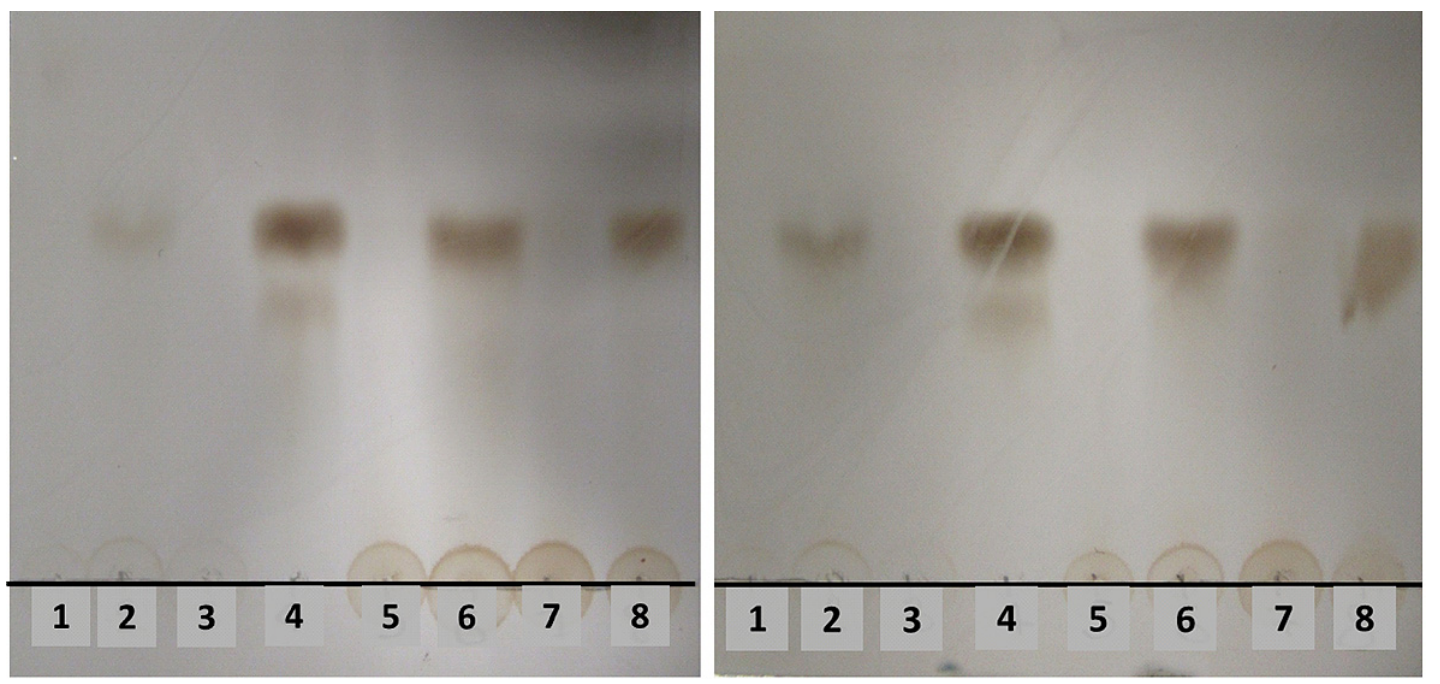

Figure 4. Thin-layer chromatogram of hydrolysis products of laminarin by crude killer toxins (KTs). Before loading, laminarin was incubated for 2 (left panel) or 4 (right panel) hours at $25{ }^{\circ} \mathrm{C}$ with:

(1) heat-inactivated Wa1F1-KT; (2) Wa1F1-KT; (3) heat-inactivated laminarinase; (4) laminarinase;

(5) heat-inactivated Wa96603-KT; (6) Wa96603-KT; (7) heat-inactivated Wm41968-KT; (8) Wm41968-KT.

\section{Discussion}

Mucosal and invasive candidiasis are the most common mycoses in humans [22-24,35,36]. Invasive candidiasis and candidemia, in particular, are an emerging health problem, especially in hospitalized and immunosuppressed individuals and patients with indwelling medical devices [22]. Additionally, the diffusion of antifungal drugs resistance in Candida spp., particularly non-albicans, makes it necessary to look for new treatments against these infections [37].

In the present study, with the aim of searching for alternative antifungal agents, a KT produced by the newly isolated $W$. anomalus $1 F 1$ strain [15] was assayed in vitro against clinical isolates and laboratory strains of $C$. albicans and C. glabrata displaying known mutations and different susceptibility to fluconazole [27-31].

KTs produced by strains of $W$. anomalus have previously shown to be active against Candida spp. both in vitro and in vivo [20,21,38,39], although little is known on their activity against resistant strains.

W. anomalus may produce different KTs with variable molecular mass $(8-300 \mathrm{kDa})$, structural characteristics, $\mathrm{pH}$ and temperature optima, and antimicrobial activity range [7,40]. KTs from $W$. anomalus and other killer yeasts have been shown to exert a $\beta$-1,3-glucanase activity, and may cause damage to the cell wall of susceptible yeasts as a result of degradation of the main $\beta$-glucans cell wall components [20,34,41,42].

Our results showed that Wa1F1-KT shares at least one epitope with the KT produced by W. anomalus ATCC 96603, although the molecular mass of the two toxins is slightly different, indicating that they are related but not identical. Both Wa96603-KT and Wa1F1-KT degraded the soluble $\beta$-glucan laminarin in a manner similar to laminarinase, an endo-1,3(4)- $\beta$-glucanase, as did Wm41968-KT, whose glucanase activity had been already suggested [34].

The spectrum of activity of Wa96603-KT and Wa1F1-KT, however, appeared to be different, as the latter proved to be active against the $C$. glabrata isolates but did not affect the majority of $C$. albicans strains tested in this study, while Wa96603-KT was previously shown to kill different clinical C. albicans isolates [43,44]. Selective killing of non-albicans species by $W$. anomalus KTs with $\beta$-glucanase activity has previously been reported [20]. This differential spectrum may be explained with differences in the specificity of $\beta$-glucanase activity of KTs, which may selectively recognize different glycosidic linkages and glucan receptors on target yeast cells $[45,46]$. 
Although some KTs from $W$. anomalus display high stability at $37{ }^{\circ} \mathrm{C}$ and even higher temperatures [47], many KTs show lower thermostability [7]. We found that the optimal temperature for Wa1F1-KT activity was lower than the physiological value in the human body. Nevertheless, the absence of $\beta$-glucans on mammalian cells suggests its potential application against fungal infections at skin and mucosal membrane levels, as has been demonstrated with other KTs [48]. Further characterization of Wa1F1-KT enzymatic activity and the cloning of its encoding gene may represent the next step to investigate the feasibility to produce molecules with broader therapeutic activity, possibly including systemic infections.

The mechanism of action of Wa1F1-KT on C. glabrata appeared to be independent from the fluconazole-resistance pathway, as only slightly different effects were observed against the susceptible clinical isolate (DSY562) or its mutant derivative strains (SFY93, SFY105, SFY115, SFY116). This phenomenon underlies the potential of Wa1F1-KT as a universally active anti-C. glabrata tool, likely not affected by drug-resistance phenotypes.

On the contrary, the fact that Wa1F1-KT was active only on C. albicans DSY289 implies that the mechanism of action of the toxin may be dependent upon the specific mutations that confer resistance to fluconazole in this strain. C. albicans DSY289 was derived from the fluconazole-susceptible clinical strain DSY347 by mutations that confer combined resistance to azoles [27,29]. In particular, the point mutations S405F/Y132H in the ERG11 gene encoding the enzyme lanosterol $14-\alpha$-sterol demethylase, which is involved in converting lanosterol into ergosterol, an essential component of the fungal cell membrane, are associated with a conformational change of the target enzyme and reduced interaction or binding of azoles [49-51]. The gain-of-function A736V mutation in the transcriptional activator $T A C 1$ causes the overexpression of the ATP binding cassette (ABC)-transporters CDR1 and CDR2, decreasing the concentration of azoles within the fungal cell $[29,52]$. It is not clear how this mutation may affect Wa1F1-KT activity, but it may be speculated that the overexpression of transport systems and associated extracellular loops could possibly alter the recognition of the cell surface target by the toxin or that the transcriptional activator $T A C 1$ is involved in the regulation of transcription of toxin receptors.

Further studies aimed at the biochemical characterization of properly purified Wa1F1-KT, the elucidation of its mechanism of action, and the reasons for its differential killing ability against different mutant strains can provide important information for developing new strategies to combat infections caused by azole-resistant Candida strains.

\section{Materials and Methods}

\subsection{Yeast Strains}

Strains belonging to the species W. anomalus, W. saturnus var. mrakii, C. lusitaniae, C. albicans, and C. glabrata were used in this study. W. anomalus $1 \mathrm{~F} 1$ isolated from P. perniciosus [15], W. anomalus ATCC 96603 (a KT-producing strain formerly referred to as UP25F) [32], and Williopsis saturnus var. mrakii MUCL 41968 [34] were used for the production of Wa1F1-KT, Wa96603-KT, and Wm41986-KT, respectively. The KT non-producing, KT-susceptible, $W$. anomalus UM3 strain was also used in this study as a negative control for KT expression [32].

The activity of Wa1F1-KT was tested against the reference C. albicans strain SC5314, two wild-type C. albicans clinical isolates (DSY544 and DSY347) [27,30], two C. albicans mutant strains resistant to fluconazole (DSY775, derived from DSY544, and DSY289, derived from DSY347) [27,29,30], two wild-type C. glabrata clinical isolates susceptible (DSY562) and resistant (DSY565) to fluconazole [28], and four C. glabrata fluconazole-resistant strains derived from DSY562 by mutations in the gene CgPDR1 (SFY93, SFY105, SFY115, SFY116) [31]. The reference strain C. lusitaniae NEQAS 6208, known to be susceptible to the activity of Wa1F1-KT [15], was also used as a positive control for KT activity.

Yeasts maintained in sterile distilled water were subcultured on Sabouraud Dextrose Agar plates. 


\subsection{Media}

KT-producing strains were grown in YPD medium (1\% yeast extract, $2 \%$ peptone, and $2 \%$ dextrose), then subcultured for KT production in YPD medium with $15 \%$ glycerol, buffered at $\mathrm{pH} 4.6$ with $0.1 \mathrm{M}$ citric acid and $0.2 \mathrm{M} \mathrm{Na}_{2} \mathrm{HPO}_{4}$. For the KT activity assay, YPD medium was added with $3 \%$ agar, and $0.003 \%$ methylene blue, and adjusted to $\mathrm{pH} 4.6$ with $0.1 \mathrm{M}$ citric acid and $0.2 \mathrm{M} \mathrm{Na}_{2} \mathrm{HPO}_{4}$.

\subsection{Production of KTs}

For the production of crude extracts, a seed culture of the $W$. anomalus and $W$. saturnus var. mrakii strains was incubated at $20{ }^{\circ} \mathrm{C}$ for $24 \mathrm{~h}$ with shaking at $150 \mathrm{rpm}$ in YPD medium. Flasks (500-mL volume) containing $100 \mathrm{~mL}$ of YPD buffered at $\mathrm{pH} 4.6$, with $15 \%$ glycerol, were inoculated with $1 \mathrm{~mL}$ of the seed culture and incubated at $20^{\circ} \mathrm{C}$ for $72 \mathrm{~h}$ with shaking ( $\left.150 \mathrm{rpm}\right)$. After this period, the cells were removed by centrifugation $\left(5000 \times g, 10 \mathrm{~min}, 4^{\circ} \mathrm{C}\right)$; the supernatant was filtered through $0.45 \mu \mathrm{m}$ pore size membranes (Merck Millipore, Darmstadt, Germany) and concentrated (50-fold) through an Amicon Ultra-15 (10-kDa cutoff) filter unit (Merck Millipore) by centrifugation at $4000 \times g$, $4{ }^{\circ} \mathrm{C}$. Accordingly, a concentrated extract of YPD medium used for KT production was prepared. The concentrated crude extracts were stored at $4{ }^{\circ} \mathrm{C}$ until use.

\subsection{Western Blot Analysis}

The crude extracts from $W$. anomalus ATCC 96603, UM3, and 1F1 were analyzed by non-continuous denaturing sodium dodecyl sulfate polyacrylamide gel electrophoresis (SDS-PAGE) in $7 \%$ polyacrylamide gel, at 100 Volts for $2 \mathrm{~h}$ in a minigel system (Bio-Rad Laboratories, Hercules, CA, USA). Concentrated YPD medium was also run as a control.

Proteins were electrically transferred to a polyvinylidene difluoride (PVDF) membrane at 100 Volts for $1 \mathrm{~h}$. The total protein content of the crude extracts was estimated by Ponceau staining prior to immunodetection. The PVDF membrane was then incubated for $1 \mathrm{~h}$ at room temperature with $5 \%$ bovine serum albumin in Tris-buffered saline (TBS) at pH 7.5 and $0.5 \%$ tween-20 (TBS-T). Subsequently, the membrane was incubated overnight at $4{ }^{\circ} \mathrm{C}$ with a 1:500 dilution in TBS-T of the monoclonal W. anomalus ATCC 96603 KT-neutralizing antibody mAbKT4 [32], known to cross-react with KTs from other Wickerhamomyces [14] and Williopsis [33] strains. After washing three times in TBS-T, the membrane was incubated for $1 \mathrm{~h}$ at room temperature with a secondary, peroxidase-conjugated anti-mouse antibody. The membrane was thoroughly washed with TBS-T, incubated for $1 \mathrm{~min}$ with the proper substrate (BM Chemiluminescence blotting substrate, Roche, Basel, Switzerland), and detected by ChemiDoc 2000R (Kodak, Rochester, NY, USA).

\subsection{Characterization of Wa1F1-KT Using Size Exclusion Chromatography}

The crude Wa1F1-KT was dialyzed against $0.01 \mathrm{M}$ citric acid- $\mathrm{Na}_{2} \mathrm{HPO}_{4}$ buffer ( $\mathrm{pH} 4.5$ ) for $24 \mathrm{~h}$ at $4{ }^{\circ} \mathrm{C}$ using a membrane with a molecular mass cut-off of $10 \mathrm{kDa}$. Analytical gel filtration was performed on a HiPrep Sephacryl S-200 prepacked column (GE Healthcare Life Sciences, Marlborough, MA, USA), characterized by bed dimensions of $16 \times 600 \mathrm{~mm}$ and an exclusion limit (for globular proteins) of about $400 \mathrm{kDa}$, connected to an AKTA purifier system (GE Healthcare Life Sciences). Dialyzed crude extract was applied to the column, equilibrated, and eluted with 1.2 column volume of citric acid- $\mathrm{Na}_{2} \mathrm{HPO}_{4}$ buffer, $\mathrm{pH}$ 4.5. Eluted fractions $(1 \mathrm{~mL})$ were combined according to chromatogram peaks, lyophilized, and re-solubilized in $1 \mathrm{~mL}$ of sterile distilled water. Total protein content was quantified with an infrared-based spectrometry system (Direct Detect ${ }^{\mathrm{TM}}$, Merck Millipore) and the concentrated fractions were assayed for killing activity against the C. lusitaniae NEQAS 6208 susceptible strain (see below). 


\subsection{Evaluation of Wa1F1-KT Activity}

The activity of Wa1F1-KT was tested against the reference C. lusitaniae NEQAS 6208 strain and fluconazole-susceptible or -resistant clinical isolates as well as laboratory strains of C. albicans and C. glabrata. Each test strain, grown overnight on SDA plates, was resuspended in water to a final concentration of $0.5 \mathrm{McFarland}$ and spread $(100 \mu \mathrm{L})$ on the surface of YPD agar plates. Crude extracts from $W$. anomalus $1 F 1$ were poured into wells of $8 \mathrm{~mm}(40 \mu \mathrm{L}$ per well) cut into the agar plates. The plates were incubated for $48 \mathrm{~h}$ at 25,30 , or $37^{\circ} \mathrm{C}$ and the diameter of the area of growth inhibition was measured.

\subsection{Laminarin Hydrolysis}

The ability of Wa1F1-KT to hydrolyze the soluble $\beta$-1,3-glucan laminarin (Sigma-Aldrich, St. Louis, MO, USA) was assayed in comparison to Wa96603-KT, Wm41968-KT (with recognized $\beta$-glucanase activity [34]), and laminarinase (Sigma-Aldrich). The reaction mixtures contained $20 \mu \mathrm{L}$ of 50 -fold concentrated crude KTs or $10 \mu \mathrm{L}$ of laminarinase $(4.5 \mathrm{U} / \mathrm{mL})$ and $2 \mathrm{mg} / \mathrm{mL}$ laminarin in $100 \mu \mathrm{L}$ of citric acid- $\mathrm{Na}_{2} \mathrm{HPO}_{4}$ buffer $(0.01 \mathrm{M}, \mathrm{pH} 4.5)$. After incubation at $25^{\circ} \mathrm{C}$ for 2 and $4 \mathrm{~h}$, the reaction was stopped by heating at $100{ }^{\circ} \mathrm{C}$ for $15 \mathrm{~min}$. The activity on laminarin was estimated through observation of the end products of laminarin hydrolysis by thin layer chromatography [53]. Reaction mixtures containing crude KTs inactivated by heating at $100{ }^{\circ} \mathrm{C}$ for $15 \mathrm{~min}$ were used as controls.

Acknowledgments: This research was supported by the Italian Ministry of Education, University, and Research program FIRB 2013 [RBFR136GFF] (to Laura Giovati and Sara Epis). The authors gratefully thank Dominique Sanglard, Institute of Microbiology, University of Lausanne, Lausanne, Suisse, for supplying C. albicans and C. glabrata DS and SF strains.

Author Contributions: L.G., S.E., and S.C. conceived and designed the experiments; L.G., C.S., E.F., and T.C. performed the experiments; L.G., E.F., S.E., and C.B. analyzed the data; E.M. and I.R. contributed materials; L.G. and S.C. wrote the paper.

Conflicts of Interest: The authors declare no conflict of interest. The founding sponsors had no role in the design of the study; in the collection, analyses, or interpretation of data; in the writing of the manuscript, and in the decision to publish the results.

\section{References}

1. Bevan, E.A.; Makower, M. The Physiological Basis of the Killer Character in Yeast; XI ${ }^{\text {th }}$ Int Congr Genet, Leiden, The Netherlands; Pergamon Press: Oxford, England, 1963; pp. 202-203.

2. Magliani, W.; Conti, S.; Gerloni, M.; Bertolotti, D.; Polonelli, L. Yeast killer systems. Clin. Microbiol. Rev. 1997, 10, 369-400. [PubMed]

3. Muccilli, S.; Restuccia, C. Bioprotective role of yeasts. Microorganisms 2015, 3, 588-611. [CrossRef] [PubMed]

4. Buzzini, P.; Turchetti, B.; Vaughan-Martini, A.E. The use of killer sensitivity patterns for biotyping yeast strains: The state of the art, potentialities and limitations. FEMS Yeast Res. 2007, 7, 749-760. [CrossRef] [PubMed]

5. Polonelli, L.; Conti, S. Biotyping of Candida albicans and other fungi by yeast killer toxins sensitivity. Methods Mol. Biol. 2009, 499, 97-115. [CrossRef] [PubMed]

6. Polonelli, L.; Magliani, W.; Ciociola, T.; Giovati, L.; Conti, S. From Pichia anomala killer toxin through killer antibodies to killer peptides for a comprehensive anti-infective strategy. Antonie Van Leeuwenhoek 2011, 99, 35-41. [CrossRef] [PubMed]

7. Walker, G.M. Pichia anomala: Cell physiology and biotechnology relative to other yeasts. Antonie Van Leeuwenhoek 2011, 99, 25-34. [CrossRef] [PubMed]

8. Valzano, M.; Cecarini, V.; Cappelli, A.; Capone, A.; Bozic, J.; Cuccioloni, M.; Epis, S.; Petrelli, D.; Angeletti, M.; Eleuteri, A.M.; et al. A yeast strain associated to Anopheles mosquitoes produces a toxin able to kill malaria parasites. Malaria J. 2016, 15, 21. [CrossRef] [PubMed]

9. Kagiyama, S.; Aiba, T.; Kadowaki, K.; Mogi, K. New killer toxins of halophilic Hansenula anomala. Agric. Biol. Chem. 1988, 52, 1-7. [CrossRef] 
10. Fredlund, E.; Druvefors, U.; Boysen, M.E.; Lingsten, K.-J.; Schnürer, J. Physiological characteristics of the biocontrol yeast Pichia anomala J121. FEMS Yeast Res. 2002, 2, 395-402. [CrossRef] [PubMed]

11. Perez, M.F.; Contreras, L.; Garnica, N.M.; Fernández-Zenoff, M.V.; Farías, M.E.; Sepulveda, M.; Ramallo, J.; Dib, J.R. Native killer yeasts as biocontrol agents of postharvest fungal diseases in lemons. PLoS ONE 2016, 11, e0165590. [CrossRef] [PubMed]

12. Wang, X.; Chi, Z.; Yue, L.; Li, J.; Li, M.; Wu, L. A marine killer yeast against the pathogenic yeast strain in crab (Portunus trituberculatus) and an optimization of the toxin production. Microbiol. Res. 2007, 162, 77-85. [CrossRef] [PubMed]

13. Ricci, I.; Damiani, C.; Scuppa, P.; Mosca, M.; Crotti, E.; Rossi, P.; Rizzi, A.; Capone, A.; Gonella, E.; Ballarini, P.; et al. The yeast Wickerhamomyces anomalus (Pichia anomala) inhabits the midgut and reproductive system of the Asian malaria vector Anopheles stephensi. Environ. Microbiol. 2011, 13, 911-921. [CrossRef] [PubMed]

14. Cappelli, A.; Ulissi, U.; Valzano, M.; Damiani, C.; Epis, S.; Gabrielli, M.G.; Conti, S.; Polonelli, L.; Bandi, C.; Favia, G.; et al. A Wickerhamomyces anomalus killer strain in the malaria vector Anopheles stephensi. PLoS ONE 2014, 9, e95988. [CrossRef] [PubMed]

15. Martin, E.; Bongiorno, G.; Giovati, L.; Montagna, M.; Crotti, E.; Damiani, C.; Gradoni, L.; Polonelli, L.; Ricci, I.; Favia, G.; et al. Isolation of a Wickerhamomyces anomalus yeast strain from the sandfly Phlebotomus perniciosus, displaying the killer phenotype. Med. Vet. Entomol. 2016, 30, 101-106. [CrossRef] [PubMed]

16. Passoth, V.; Fredlund, E.; Druvefors, U.Ä.; Schnürer, J. Biotechnology, physiology and genetics of the yeast Pichia anomala. FEMS Yeast Res. 2006, 6, 3-13. [CrossRef] [PubMed]

17. De Hoog, G.S. Risk assessment of fungi reported from humans and animals. Mycoses 1996, 39, $407-417$. [CrossRef] [PubMed]

18. Epis, S.; Capone, A.; Martin, E.; Paolucci, M.; Bazzocchi, C.; Valzano, M.; Bozic, J.; Novati, S.; Favia, G.; Ricci, I. A rapid qPCR method to investigate the circulation of the yeast Wickerhamomyces anomalus in humans. New Microbiol. 2015, 38, 577-581. [PubMed]

19. İzgü, F.; Altınbay, D.; Türeli, A.E. In vitro activity of panomycocin, a novel exo- $\beta$-1,3-glucanase isolated from Pichia anomala NCYC 434, against dermatophytes. Mycoses 2007, 50, 31-34. [CrossRef] [PubMed]

20. Tay, S.-T.; Lim, S.-L.; Tan, H.-W. Growth inhibition of Candida species by Wickerhamomyces anomalus mycocin and a lactone compound of Aureobasidium pullulans. BMC Complement. Altern. Med. 2014, 14, 439. [CrossRef] [PubMed]

21. Paris, A.P.; Persel, C.; Serafin, C.F.; de Cassia Garcia Simao, R.; Gandra, R.F. Susceptibility of Candida albicans isolated from blood to Wickerhamomyces anomalous mycocins. Curr. Microbiol. 2016, 73, 878-884. [CrossRef] [PubMed]

22. Enoch, D.A.; Yang, H.; Aliyu, S.H.; Micallef, C. The changing epidemiology of invasive fungal infections. In Human Fungal Pathogen Identification: Methods and Protocols; Lion, T., Ed.; Springer: New York, NY, USA, 2017; pp. 17-65.

23. Guinea, J. Global trends in the distribution of Candida species causing candidemia. Clin. Microbiol. Infect. 2014, 20, 5-10. [CrossRef] [PubMed]

24. Lockhart, S.R. Current epidemiology of Candida infection. Clin. Microbiol. Newsl. 2014, 36, 131-136. [CrossRef]

25. Orasch, C.; Marchetti, O.; Garbino, J.; Schrenzel, J.; Zimmerli, S.; Mühlethaler, K.; Pfyffer, G.; Ruef, C.; Fehr, J.; Zbinden, R.; et al. Candida species distribution and antifungal susceptibility testing according to European Committee on Antimicrobial Susceptibility Testing and new vs. old Clinical and Laboratory Standards Institute clinical breakpoints: A 6-year prospective candidaemia survey from the fungal infection network of Switzerland. Clin. Microbiol. Infect. 2014, 20, 698-705. [CrossRef] [PubMed]

26. Tan, T.Y.; Hsu, L.Y.; Alejandria, M.M.; Chaiwarith, R.; Chinniah, T.; Chayakulkeeree, M.; Choudhury, S.; Chen, Y.H.; Shin, J.H.; Kiratisin, P.; et al. Antifungal susceptibility of invasive Candida bloodstream isolates from the Asia-Pacific region. Med. Mycol. 2016, 54, 471-477. [CrossRef] [PubMed]

27. Sanglard, D.; Kuchler, K.; Ischer, F.; Pagani, J.L.; Monod, M.; Bille, J. Mechanisms of resistance to azole antifungal agents in Candida albicans isolates from AIDS patients involve specific multidrug transporters. Antimicrob. Agents Chemother. 1995, 39, 2378-2386. [CrossRef] [PubMed]

28. Sanglard, D.; Ischer, F.; Calabrese, D.; Majcherczyk, P.A.; Bille, J. The ATP binding cassette transporter gene CgCDR1 from Candida glabrata is involved in the resistance of clinical isolates to azole antifungal agents. Antimicrob. Agents Chemother. 1999, 43, 2753-2765. [PubMed] 
29. Coste, A.; Selmecki, A.; Forche, A.; Diogo, D.; Bougnoux, M.-E.; d’Enfert, C.; Berman, J.; Sanglard, D. Genotypic evolution of azole resistance mechanisms in sequential Candida albicans isolates. Eukaryot. Cell 2007, 6, 1889-1904. [CrossRef] [PubMed]

30. Coste, A.T.; Crittin, J.; Bauser, C.; Rohde, B.; Sanglard, D. Functional analysis of cis- and trans-acting elements of the Candida albicans CDR2 promoter with a novel promoter reporter system. Eukaryot. Cell 2009, 8, 1250-1267. [CrossRef] [PubMed]

31. Ferrari, S.; Ischer, F.; Calabrese, D.; Posteraro, B.; Sanguinetti, M.; Fadda, G.; Rohde, B.; Bauser, C.; Bader, O.; Sanglard, D. Gain of function mutations in CgPDR1 of Candida glabrata not only mediate antifungal resistance but also enhance virulence. PLoS Pathog. 2009, 5, e1000268. [CrossRef] [PubMed]

32. Polonelli, L.; Séguy, N.; Conti, S.; Gerloni, M.; Bertolotti, D.; Cantelli, C.; Magliani, W.; Cailliez, J.C. Monoclonal yeast killer toxin-like candidacidal anti-idiotypic antibodies. Clin. Diagn. Lab. Immunol. 1997, 4, 142-146. [PubMed]

33. Guyard, C.; Evrard, P.; Corbisier-Colson, A.M.; Louvart, H.; Dei-Cas, E.; Menozzi, F.D.; Polonelli, L.; Cailliez, J. Immuno-crossreactivity of an anti-Pichia anomala killer toxin monoclonal antibody with a Williopsis saturnus var. mrakii killer toxin. Med. Mycol. 2001, 39, 395-400. [CrossRef] [PubMed]

34. Guyard, C.; Dehecq, E.; Tissier, J.-P.; Polonelli, L.; Dei-Cas, E.; Cailliez, J.-C.; Menozzi, F.D. Involvement of beta-glucans in the wide-spectrum antimicrobial activity of Williopsis saturnus var. mrakii MUCL 41968 killer toxin. Mol. Med. 2002, 8, 686-694. [PubMed]

35. López-Martínez, R. Candidosis, a new challenge. Clin. Dermatol. 2010, 28, 178-184. [CrossRef] [PubMed]

36. Pfaller, M.A.; Diekema, D.J. Epidemiology of invasive candidiasis: A persistent public health problem. Clin. Microbiol. Rev. 2007, 20, 133-163. [CrossRef] [PubMed]

37. Spampinato, C.; Leonardi, D. Candida infections, causes, targets, and resistance mechanisms: Traditional and alternative antifungal agents. Biomed. Res. Int. 2013, 2013, 204237. [CrossRef] [PubMed]

38. Polonelli, L.; Archibusacci, C.; Sestito, M.; Morace, G. Killer system: A simple method for differentiating Candida albicans strains. J. Clin. Microbiol. 1983, 17, 774-780. [PubMed]

39. Polonelli, L.; Conti, S.; Gerloni, M.; Campani, L.; Mantovani, M.P.; Morace, G. Production of yeast killer toxin in experimentally infected animals. Mycopathologia 1990, 110, 169-175. [CrossRef] [PubMed]

40. Guo, F.-J.; Ma, Y.; Xu, H.-M.; Wang, X.-H.; Chi, Z.-M. A novel killer toxin produced by the marine-derived yeast Wickerhamomyces anomalus YF07b. Antonie Van Leeuwenhoek 2013, 103, 737-746. [CrossRef] [PubMed]

41. İzgü, F.; Altınbay, D. Isolation and characterization of the K5-type yeast killer protein and its homology with an exo-beta-1,3-glucanase. Biosci. Biotechnol. Biochem. 2004, 68, 685-693. [CrossRef] [PubMed]

42. Wang, L.; Yue, L.; Chi, Z.; Wang, X. Marine killer yeasts active against a yeast strain pathogenic to crab Portunus trituberculatus. Dis. Aquat. Organ. 2008, 80, 211-218. [CrossRef] [PubMed]

43. Mathews, H.L.; Conti, S.; Witek-Janusek, L.; Polonelli, L. Effect of Pichia anomala killer toxin on Candida albicans. Med. Mycol. 1998, 36, 199-204. [CrossRef] [PubMed]

44. Polonelli, L.; Beninati, C.; Teti, G.; Felici, F.; Ciociola, T.; Giovati, L.; Sperindè, M.; Passo, C.L.; Pernice, I.; Domina, M.; et al. Yeast killer toxin-like candidacidal Ab6 antibodies elicited through the manipulation of the idiotypic cascade. PLoS ONE 2014, 9, e105727. [CrossRef] [PubMed]

45. Gopal, P.; Sullivan, P.A.; Shepherd, M.G. Isolation and structure of glucan from regenerating spheroplasts of Candida albicans. Microbiology 1984, 130, 1217-1225. [CrossRef] [PubMed]

46. İzgü, F.; Altınbay, D.; Acun, T. Killer toxin of Pichia anomala NCYC 432; purification, characterization and its exo- $\beta$-1,3-glucanase activity. Enzyme Microb. Technol. 2006, 39, 669-676. [CrossRef]

47. Chi, Z.; Chi, Z.; Zhang, T.; Liu, G.; Li, J.; Wang, X. Production, characterization and gene cloning of the extracellular enzymes from the marine-derived yeasts and their potential applications. Biotechnol. Adv. 2009, 27, 236-255. [CrossRef] [PubMed]

48. Polonelli, L.; Lorenzini, R.; De Bernardis, F.; Morace, G. Potential therapeutic effect of yeast killer toxin. Mycopathologia 1986, 96, 103-107. [CrossRef] [PubMed]

49. Franz, R.; Kelly, S.L.; Lamb, D.C.; Kelly, D.E.; Ruhnke, M.; Morschhäuser, J. Multiple molecular mechanisms contribute to a stepwise development of fluconazole resistance in clinical Candida albicans strains. Antimicrob. Agents Chemother. 1998, 42, 3065-3072. [PubMed]

50. Kanafani, Z.A.; Perfect, J.R. Resistance to antifungal agents: Mechanisms and clinical impact. Clin. Infect. Dis. 2008, 46, 120-128. [CrossRef] [PubMed] 
51. Xu, Y.; Sheng, F.; Zhao, J.; Chen, L.; Li, C. ERG11 mutations and expression of resistance genes in fluconazole-resistant Candida albicans isolates. Arch. Microbiol. 2015, 197, 1087-1093. [CrossRef] [PubMed]

52. Coste, A.T.; Karababa, M.; Ischer, F.; Bille, J.; Sanglard, D. TAC1, transcriptional activator of CDR genes, is a new transcription factor involved in the regulation of Candida albicans ABC transporters CDR1 and CDR2. Eukaryot. Cell 2004, 3, 1639-1652. [CrossRef] [PubMed]

53. Gong, F.; Sheng, J.; Chi, Z.; Li, J. Inulinase production by a marine yeast Pichia guilliermondii and inulin hydrolysis by the crude inulinase. J. Ind. Microbiol. Biotechnol. 2007, 34, 179-185. [CrossRef] [PubMed] 